\title{
All-optical pulse bursts generation from a nonlinear amplifying loop mirror
}

\author{
Auro M. Perego ${ }^{1, *}$ \\ ${ }^{1}$ Aston Institute of Photonics Technologies, Aston University, Aston Triangle, Birmingham B4 7ET, UK. \\ *a.perego1@aston.ac.uk \\ Compiled May 18, 2019
}

A novel method for the generation of bursts of optical pulses is proposed. It is shown analytically that a nonlinear amplifying loop mirror in single pass configuration can transform a low power input pulse into a burst consisting of pulses with individual energy up to tens of nJ. The burst features, number of pulses, their peak power, energy and duration can be tuned and controlled. Numerical simulations show robustness of the technique to presence of Raman scattering and that subpicosencod pulse duration can be achieved. The latter highlights the relevance of the proposed pulse bursts generator for material processing and in medical applications involving optical ablation. (๑) 2019 Optical Society of America

OCIS codes: (140.3390) Laser materials processing, (320.5540) Pulse shaping, (170.1020) Ablation of tissue.

http://dx.doi.org/10.1364/ao.XX.XXXXXX

\section{INTRODUCTION}

The nonlinear loop mirror (NOLM) is a photonics technology device invented by Doran and Wood in 1999 with remarkable properties as a nonlinear optical switch[1]. It consists in an optical fibre loop where light can be injected and extracted in correspondence to a beam splitter (coupler). Light is injected in correspondence to the coupler which splits the input pulse into two waves; the two waves propagate in opposite directions through the fiber loop and recombine then again at the coupler. If the coupler splitting ratio is unbalanced (i.e. different from 50:50), the asymmetric self-phase modulation suffered by the two counterpropagating waves will results in a non trivial power dependent transmission function at the loop mirror output: the transmission function is characterized by a series of minima and maxima. It follows that a natural application of NOLM is signal regeneration: due to its power dependent transmission function it can be used to suppress undesired low power features (e.g. tails) of optical pulses and temporal solitons hence behaving as an all-optical signal regenerator both for on-off-keyed signals[2], but also in multilevel regeneration applications[3]. Beside the regeneration application, NOLM can be inserted in a laser cavity to perform the role of an effective saturable absorber allowing mode-locking operation[4,5]. A natural extension of the NOLM is the nonlinear amplifying loop mirror (NALM). The latter was proposed by Fermann and co-authors in 1990 [6] and differs from the NOLM by the introduction of an amplifier inside the fiber loop, in general located just after the coupler. The presence of the amplifier allows to achieve an asymmetry in the nonlinear effects suffered by the two counterpropagating fields even for balanced coupler splitting ratio. The NALM has hence become a popular tool for signal regeneration and as a laser mode-locker too [7]. Both in case of signal regeneration and when used as saturable absorbers NOLM and NALM perform the task of suppressing noise and enhancing the visibility of certain optical signals or pulses.

With the exception of a few studies $[8,9]$ the opposite problem of using NOLM or NALM to break-up very clean input waveforms has not been thoroughly investigated nor its impact for applications has been considered. The aim of the present letter is to show how the nontrivial multi-peak transmission function of the NALM could be used to generate pulse bursts and to suggest hence new potential applications of the NALM in photonics technologies. To properly motivate the practical interest of the present study it proves useful to introduce some of the emerging trends in photonic based material processing.

Material processing is one of the numerous successful applications of laser technology. In particular, in this context lasers are used for instance to drill holes, cut or melt materials and for ablation. In the case of ablation the high intensity coherent radiation, emitted by a laser and incident on a material is able to melt the upper layers of the surface and eventually to remove them [10-12]. For the process to take place a large amount of energy should impinge on the material, in order to reach a critical temperature necessary to trigger ablation. One drawback of this technique is that, once a highly energetic pulse has hit the material causing the removal of part of its surface, then the sample temperature drops due to heat dissipated with the large portion of removed material. Such temperature drop makes the ablation process inefficient. Kerse and co-authors [12] have suggested to use lasers operating in burst mode so that heat is dissipated from the material at slower rate, hence without reducing the ablation process efficiency. They showed that indeed a burst of pulses is much more efficient in ablating the target material than a single pulse with the same energy. Hence the material sample to be processed in such ablation cooling regime is illuminated not by a regular train of light pulses, but by a series of low repetition rate $(\mathrm{kHz} / \mathrm{MHz})$ bursts each one consisting of a limited number 
of pulses (from a few tens to a few hundreds).

A serious effort is being undertaken in order to find efficient and reasonably simple ways to generate bursts of light pulses [13-18]. It is worth stressing that the existing solutions are rather bulky and complicated relying on several amplification stages, dispersion compensation, compression and on electronics (modulators, wave-form generators) for pulse shaping. It is hence of great applied interest the possibility of designing a compact all-optical system able to generate bursts of pulses from commercially available laser sources without the use of complicated pulse shaping techniques and without relying on electronics. In this Letter a simple scheme is proposed to generate bursts of light pulses with tuneable features based on a NALM in the single pass operational regime. The approach is simple and effective, relying on independent control of the bursts parameters.

\section{CONCEPT AND SETUP}

The bursts generator is very simple: it consists in a standard pulsed laser source pumping one input arm of a NALM. The generic pump laser provides a seed pulse whereas the NALM provides both amplification and the asymmetry needed to have a non trivial transmission function exhibiting alternating maxima and minima for several different values of the intensity input. Since the power of the input pulse varies continuously from zero to its maximum and then again to zero across the temporal pulse profile, different parts of the pulse will have different reflection and transmission properties. Temporally contiguous pulse portions will be reflected and transmitted by the loop mirror in alternating fashion. The input pulse injected into the NALM will be hence amplified and then partially reflected -partially transmitted. Both the reflected and the transmitted waves will consist in a burst of shorter pulses. The proposed setup is illustrated schematically in Fig.1. The NALM transmission function,

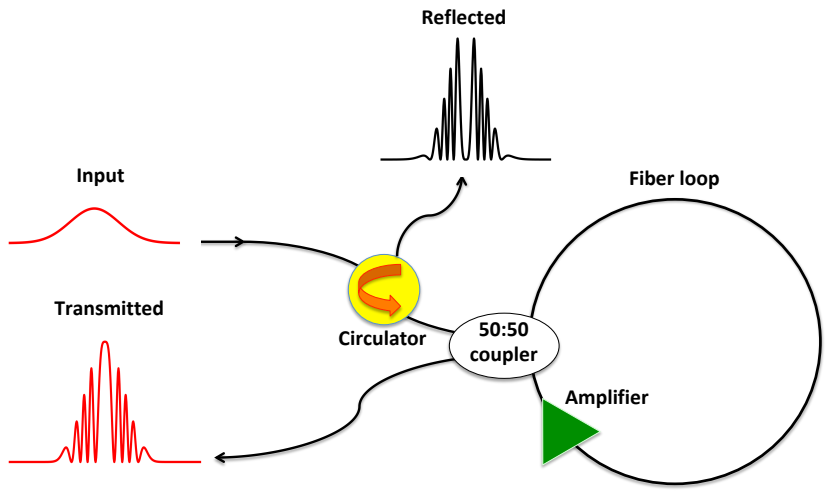

Fig. 1. Setup schematic: a low power input pulse is injected into a NALM, made by a normal dispersion fibre (large black circle) and a powerful amplifier (green triangle) located close to the 50:50 coupler. The transmitted power consists in a high energy burst of pulses, the reflected power is a burst whose pulses have peaks located in correspondence to the zeros of the transmitted power and can be extracted using a circulator (yellow circle with curved arrow).

relating the input $P_{\text {in }}(t)$ with the output power $P_{\text {out }}(t)$, for a balanced 50:50 coupler, assuming no dispersion nor distributed losses in the fiber loop, can be calculated analytically and reads:

$$
P_{\text {out }}(t)=P_{\text {in }}(t) G\left\{\frac{1}{2}+\frac{1}{2} \cos \left(\left[\frac{G-1}{2}\right] \gamma P_{\text {in }}(t) L\right)\right\}
$$

where $\gamma$ is the fibre nonlinearity coefficient, $G$ is the amplifier power gain. Maxima and minima of the transmission function are defined by the following relation

$$
\frac{G-1}{2} \gamma P_{\text {in }}(t) L=n \pi, \quad n \in \mathbb{N}
$$

When $n$ is odd the transmission function has a minimum, on the contrary when $n$ is even a maximum occurs. Hence for a given input pulse, sub-pulses are "carved out" of the input envelope as the intensity rises (falls) along the edges of the input pulse cycle through the multiple transmission peaks of the NALM. Sub-pulses are each generated twice and symmetrically, on the rising edge and on the falling edge of the input pulse. To ensure the splitting of a low power input pulse into a burst with many sub-pulses, and hence multiple oscillations of the NALM transmission function, a large gain is required: a powerful amplifier providing from 30 to $40 \mathrm{~dB}$ gain would be desired. In this respect, Ytterbium amplifier with very high saturation power exceeding $100 \mathrm{~W}$ would be excellent candidates [19]. The fibre loop should have normal dispersion to avoid modulation instability and as long as the generated pulses are longer than a few ps, dispersion can be disregarded and in this case Eqs.1,2 provide an excellent description of the NALM. The presence of the circulator in the reflected branch of the NALM, allows to extract the reflected bursts too. It is possible to tune the inter burst repetition rate by varying the single pulse repetition rate of the pump source. The proposed method is all-optical and does not require the support of any wave-form generator or modulator as typically used in previous burst pulses sources. As the source for the input pulse a standard mode-locked laser, but also a single frequency modulated diode laser could be used. Across the whole paper an input Gaussian pulse has been considered. More precisely the pulse field envelope has been defined as a function of time $t$ by the following expression: $A_{\text {in }}(t)=\sqrt{P} e^{-t^{2} / \sigma^{2}}$, where $\sigma$ is proportional to the pulse duration and $P$ is its peak power, while $P_{i n}$ is defined accordingly as $P_{i n}(t)=\left|A_{\text {in }}(t)\right|^{2}$. First an input pulse with Gaussian profile of about 4 ns FWHM duration and peak power of $10 \mathrm{~mW}$ has been considered in this case analytical results can be obtained based on Eqs.1,2. Subsequently, in order to demonstrate the reliability of the method in a more realistic settings to provide bursts with sub-picosecond pulses, numerical simulations based on a generalized nonlinear Schrödinger equation have been performed successfully proving the robustness of the technique.

\section{ANALYTICAL RESULTS}

According to Eq.1 an input pulse with the features just described above is transformed, at the NALM output, into two bursts of pulses (one reflected, the other one transmitted). Each burst may contain a number of pulses varying from less than 10 to more than 50 depending on the amplifier gain and on the fiber loop length and nonlinearity. 

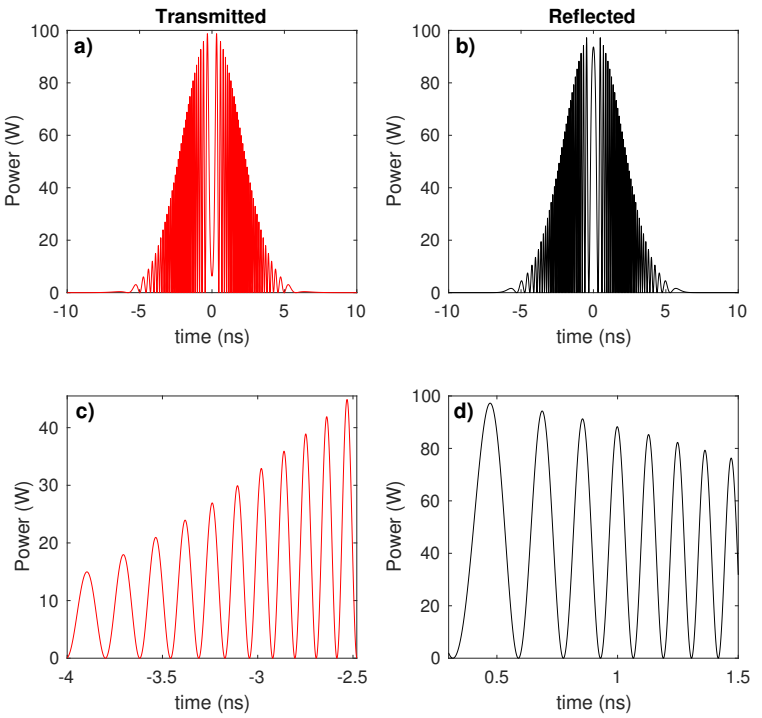

Fig. 2. Examples of the burst generator output. In a) the transmitted output for $\gamma=14 \mathrm{~W}^{-1} \mathrm{~km}^{-1}, L=0.3 \mathrm{~km}$ and $G$ corresponding to $40 \mathrm{~dB}$ gain while in $\mathrm{b}$ ) the reflected output is plotted. In c) and d) zooms of a) and b) respectively are depicted. Parameters of the input pulse are $\sigma=4 \mathrm{~ns}$ and $P=0.01$ W.

Figure 2 shows the transmitted and reflected power consisting in a burst of short pulses, being each pulse a few tens of ps long. The energy content of pulses located close to centre of the burst peak is about $10 \mathrm{~nJ}$ while close to the burst tails is about 1 nJ. In Fig.3 the tuneability of the system in term of number of pulses generated per burst has been demonstrated by showing that a low number of pulses can be achieved by simply varying the amplifier gain in the NALM. For lower amplifier gain we observe an increase in the pulses duration and a decrease of their peak power. Such feature could be hence used to tailor the burst properties depending on the required application. By keeping instead a fixed amplifier gain but using a smaller value of the loop mirror nonlinearity coefficient $\gamma$ the number of generated pulses is lowered and their energy content results increased reaching a few tens of nJ. The number of pulses generated by the NALM
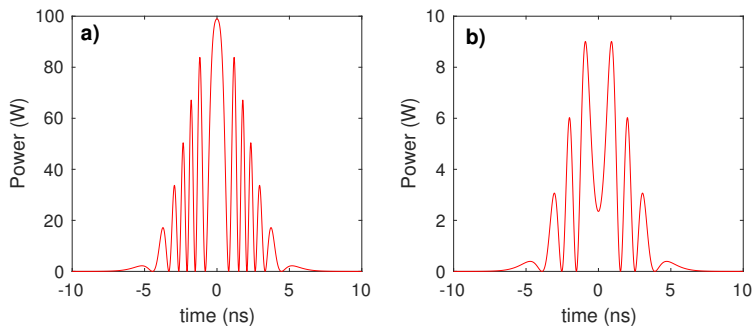

Fig. 3. Tuneability of bursts features with respect to fiber loop nonlinearity and amplifier gain. In a) $\gamma=2.5 \mathrm{~W}^{-1} \mathrm{~km}^{-1}$ has been used, all other parameters are as in Fig. 2. In b) $30 \mathrm{~dB}$ gain for the amplifier has been considered, all other parameters are as in Fig. 2.

increases, as one can expect from Fig.1, for larger amplifier gain. This fact can be appreciated graphically from Fig.4

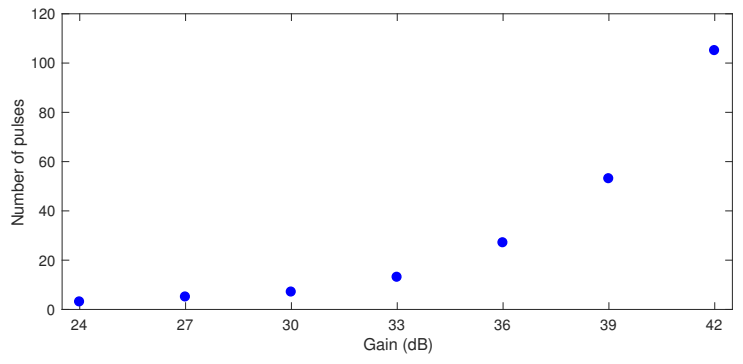

Fig. 4. The dependence of the number of pulses is plotted versus amplifier power gain in $\mathrm{dB}$. Parameters used are $\gamma=14$ $\mathrm{km}^{-1}, L=0.3 \mathrm{~km}, \sigma=4 \mathrm{~ns}, P=0.01 \mathrm{~mW}$.

\section{NUMERICAL SIMULATIONS RESULTS}

In order to provide an insight into the potentialities of the proposed techniques for optical ablation, where pulses of subpicosecond duration are required[12], the analytical approach is limited and numerical simulations are needed. This is due to the fact that, for such durations, the loop mirror dispersion can not be disregarded anymore and furthermore Raman scattering could play a role[20]. In order to numerically simulate the burst generation, the following procedure has been used. From the input pulse the clockwise and counterclockwise propagating pulses in the loop mirror are defined in the following way:

$$
\begin{aligned}
& A_{1}=A_{i n} \sqrt{\frac{1}{2}} \\
& A_{2}=A_{i n} i \sqrt{\frac{1}{2}}
\end{aligned}
$$

then the counterclockwise propagating envelope $A_{2}$ is multiplied by a positive real number $g$ corresponding to the desired amplifier gain in $\mathrm{dB}$, and then its propagation is simulated based on a generalized nonlinear Schrödinger equation. For the clockwise propagating field the propagation through the fiber is instead is calculated before multiplication with the amplification factor. Then the reflected, $A_{t}$, and transmitted, $A_{r}$, amplitudes are computed as follows:

$$
\begin{aligned}
& A_{t}=\bar{A}_{2} \sqrt{\frac{1}{2}}-i \bar{A}_{1} \sqrt{\frac{1}{2}} \\
& A_{r}=\bar{A}_{1} \sqrt{\frac{1}{2}}-i \bar{A}_{2} \sqrt{\frac{1}{2}}
\end{aligned}
$$

where $\bar{A}_{1,2}$, are the clockwise and anticlockwise travelling amplitudes after fiber loop propagation respectively. In the fiber loop, the evolution along the spatial coordinate $z$ of both fields amplitudes $A_{1}$ and $A_{2}$ defined in the comoving temporal reference frame of coordinate $t$, is described by the following generalized nonlinear Schrödinger equation [21] :

$$
\begin{aligned}
& \frac{\partial A_{1,2}}{\partial z}=-i \frac{\beta_{2}}{2} \frac{\partial^{2} A_{1,2}}{\partial^{2} t}+\frac{\beta_{3}}{6} \frac{\partial^{3} A_{1,2}}{\partial t^{3}}-\frac{\alpha}{2} A_{1,2} \\
& +i \gamma\left(\left|A_{1,2}\right|^{2} A_{1,2}+\frac{i}{\omega_{0}} \frac{\partial\left(\left|A_{1,2}\right|^{2} A_{1,2}\right)}{\partial t}-T_{R} A_{1,2} \frac{\partial\left|A_{1,2}\right|^{2}}{\partial t}\right)
\end{aligned}
$$

Eq.5 which includes the group velocity dispersion with coefficient $\beta_{2}$, third order dispersion $\beta_{3}$, losses with coefficient $\alpha$ and Raman scattering effects through the well known shock and self-steepening terms where $\omega_{0}$ is the carrier frequency and $T_{R}$ 
depends on the slope of the Raman gain spectrum. Such approximation of the Raman delayed response function is indeed valid for pulses of duration of a few hundreds of fs and longer [21]. For those simulations an about 8 ps long input pulse has been considered. An example of generated burst containing more than 50 pulses is shown in Fig.5. The sub-pulses have a duration of the order of $0.1 \mathrm{ps}$. Such short pulse duration is compatible with experimental values reported in reference[12] where pulses from $300 \mathrm{fs}$ to $1 \mathrm{ps}$ long were employed. It is interesting to notice how the pulse burst obtained in numerical simulations exhibits the typical qualitative shape of the one predicted in theory, with an extra slight asymmetry. This shows the potential robustness of the burst generator, however it is likely that for even shorter pulse duration or larger values of $\beta_{3}$, the interplay between Raman effect and third order dispersion may lead to a destabilization of the pulses. It has been furthermore verified that using a chirped input pulse does not affect the performances of the burst generator.
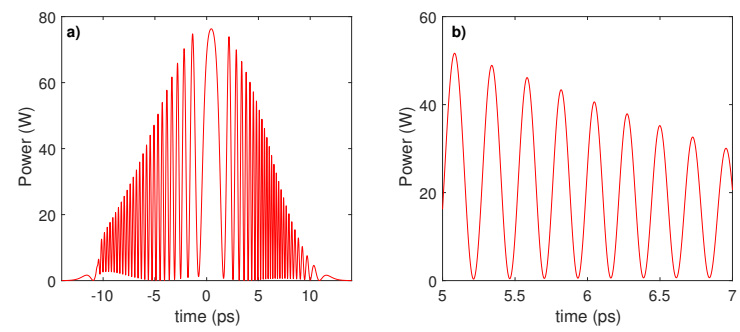

Fig. 5. Burst of pulses obtained from numerical simulations of Eq.5 are shown in a), a zoom of the pulses is shown in b). Parameters used are: $\beta_{2}=1 \mathrm{ps}^{2} / \mathrm{km}, \beta_{3}=0.12 \mathrm{ps}^{3} / \mathrm{km}, \gamma=14$ $\mathrm{km}^{-1} \mathrm{~W}^{-1}, \alpha=0.05 \mathrm{~km}^{-1}, \mathrm{~T}_{R}=3 \mathrm{fs}, \omega_{0}=1.2161 \cdot 10^{6} \mathrm{rad} / \mathrm{ns}$, $P=0.01 \mathrm{~W}, \sigma=8$ ps.

An even more realistic description of the burst formation process would require including amplifier saturation effects (both within the same pulse and between waves propagating clockwise and counterclockwise respectively, to understand whether the clockwise propagating pulse may see the amplifier saturated by the counter clockwise propagating one) and polarization effects $[20,22]$. Concerning the impact of polarization, results presented in [22] where the interplay of self-phase modulation, cross-phase modulation and random birefringence was considered, may suggest the possibility of a polarization insensitive operational mode of the NALM based burst generator too. The possibility of using polarization maintaining fiber and amplifier in the loop mirror could be considered as well. Those deeper investigations go beyond the scope of this Letter and will be addressed in future works. Furthermore it would be interesting to investigate experimentally which are the advantages and disadvantages of the presented NALM burst features compared to other existing techniques[15] for the specific application of optical ablation. One current limitation of the proposed technique, which could be overcome with new progresses in high power amplifiers technology, is the requirement of a very powerful amplifier capable to provide around $40 \mathrm{~dB}$ gain for generating bursts whose constitutive pulses have tens of nJ energy content starting from an input pulse with few $\mathrm{mW}$ peak power. Such high gain is needed to achieve the multiple oscillations of the NALM transmission function, but the latter could alternatively be achieved by increasing the fiber loop length as it can be seen from Eq.1. In this case, however the impact of dispersion should be evaluated.

\section{CONCLUSIONS}

In conclusion, in this Letter it has been shown how to exploit the non trivial NALM transmission function in order to create a single pass photonic device suitable for the generation of bursts of optical pulses with potentially sup-picosecond individual pulse duration. The system is simple, just requiring a standard mode-locked laser source NALM with normal dispersion fibre endowed with a powerful amplifier. The generated bursts of pulses may find natural applications in ablation both for material processing and for medical applications, but could possibly be used in the future in other contexts such as micromachining[23], free electron laser injection[24] and flow measurements in fluids $[25,26]$.

\section{REFERENCES}

1. N. J. Doran and D. Wood, Opt. Lett. 13, 56 (1988).

2. S. Boscolo, S. K. Turitsyn, and K. J. Blow, Opt. Fiber Technol. 14, 299-316 (2008)

3. F. Wen, C. P. Tsekrekos, Y. Geng, X. Zhou, B. Wu, K. Qiu, S. K. Turitsyn, and S. Sygletos, Opt. Express 26, 12698 (2018).

4. I. N. Duling, C. . Chen, P. K. A. Wai, and C. R. Menyuk, IEEE J. Quantum Electron. 30, 194 (1994).

5. I. N. Duling, Opt. Lett. 16, 539 (1991).

6. M. E. Fermann, F. Haberl, M. Hofer, and H. Hochreiter, Opt. Lett. 15, 752 (1990).

7. C. Aguergaray, N. G. R. Broderick, M. Erkintalo, J. S. Y. Chen, and V. Kruglov, Opt. Express 20, 10545 (2012).

8. M. Bello-Jiménez, E. A. Kuzin, O. Pottiez, B. Ibarra-Escamilla, A. FloresRosas, and M. Durán-Sánchez, J. Opt. Soc. Am. B 26, 1456 (2009).

9. M. Bello-Jiménez, E. A. Kuzin, O. Pottiez, B. Ibarra-Escamilla, A. FloresRosas, and M. Durán-Sánchez, Opt. Express 18, 2090 (2010).

10. B. N. Chichkov, C. Momma, S. Nolte, F. von Alvensleben, and A. Tünnermann, Appl. Phys. A 63, 109 (1996).

11. M. Lapczyna, K. Chen, P. Herman, H. Tan, and R. Marjoribanks, Appl. Phys. A 69, S883 (1999).

12. C. Kerse et al., Nature 537, 84-88 (2016).

13. H. Kalayciouglu, Y. B. Eldeniz, O. Akcaalan, S. Yavas, K. Gürel, M. Efe, and F. O. Ilday, Opt. Lett. 37, 2586 (2012).

14. H. Kalaycioglu, K. Eken, and F. O. Ilday, Opt. Lett. 36, 3383 (2011).

15. H. Kalaycioglu, P. Elahi, O. Akcaalan, and F. O. Ilday, IEEE J. Sel. Top. Quantum Electron. 24, 1 (2018).

16. S. Breitkopf, A. Klenke, T. Gottschall, H.-J. Otto, C. Jauregui, J. Limpert, and A. Tünnermann, Opt. Lett. 37, 5169 (2012).

17. H. Yu, J. Zhang, Y. Qi, L. Zhang, S. Zou, L. Wang, and X. Lin, J. Light. Technol. 33, 1761 (2015).

18. N. Jiang, M. C. Webster, and W. R. Lempert, Appl. Opt. 48, B23 (2009).

19. P. Wang and W. A. Clarkson, Opt. Lett. 32, 2605 (2007).

20. N. Finlayson, B. K. Nayar, and N. J. Doran, Opt. Lett. 17, 112 (1992).

21. G. P. Agrawal, Nonlinear fiber optics (Academic Press, San Diego, 2007).

22. C. B. Clausen, J. H. Povlsen, and K. Rottwitt, Opt. Lett. 21, 1535 (1996).

23. R. R. Gattass, L. R. Cerami, and E. Mazur, Opt. Express 14, 5279 (2006).

24. I. Will, H. I. Templin, S. Schreiber, and W. Sandner, Opt. Express 19, 23770 (2011).

25. B. Thurow, J. Hileman, W. Lempert, and M. Samimy, Phys. Fluids 14, 3449 (2002).

26. P. Wu, W. L. Lempert, and R. B. Miles, AIAA J. 38, 672 (2000). 


\section{FULL REFERENCES}

1. N. J. Doran and D. Wood, "Nonlinear-optical loop mirror," Opt. Lett. 13, 56-58 (1988).

2. S. Boscolo, S. K. Turitsyn, and K. J. Blow, "Nonlinear loop mirror-based all-optical signal processing in fiber-optic communications," Opt. Fiber Technol. 14, 299-316 (2008).

3. F. Wen, C. P. Tsekrekos, Y. Geng, X. Zhou, B. Wu, K. Qiu, S. K. Turitsyn, and S. Sygletos, "All-optical multilevel amplitude regeneration in a single nonlinear optical loop mirror," Opt. Express 26, 12698-12706 (2018).

4. I. N. Duling, C. . Chen, P. K. A. Wai, and C. R. Menyuk, "Operation of a nonlinear loop mirror in a laser cavity," IEEE J. Quantum Electron. 30, 194-199 (1994).

5. I. N. Duling, "All-fiber ring soliton laser mode locked with a nonlinear mirror," Opt. Lett. 16, 539-541 (1991).

6. M. E. Fermann, F. Haberl, M. Hofer, and H. Hochreiter, "Nonlinear amplifying loop mirror," Opt. Lett. 15, 752-754 (1990).

7. C. Aguergaray, N. G. R. Broderick, M. Erkintalo, J. S. Y. Chen, and V. Kruglov, "Mode-locked femtosecond all-normal all-pm yb-doped fiber laser using a nonlinear amplifying loop mirror," Opt. Express 20, 10545-10551 (2012).

8. M. Bello-Jiménez, E. A. Kuzin, O. Pottiez, B. Ibarra-Escamilla, A. FloresRosas, and M. Durán-Sánchez, "Soliton extraction from a bunch of solitons resulting from pulse breakup by using a nonlinear optical loop mirror," J. Opt. Soc. Am. B 26, 1456-1462 (2009).

9. M. Bello-Jiménez, E. A. Kuzin, O. Pottiez, B. Ibarra-Escamilla, A. FloresRosas, and M. Durán-Sánchez, "Experimental investigation of the extraction of solitons at the initial stage of the soliton formation process," Opt. Express 18, 2090-2099 (2010).

10. B. N. Chichkov, C. Momma, S. Nolte, F. von Alvensleben, and A. Tünnermann, "Femtosecond, picosecond and nanosecond laser ablation of solids," Appl. Phys. A 63, 109-115 (1996).

11. M. Lapczyna, K. Chen, P. Herman, H. Tan, and R. Marjoribanks, "Ultra high repetition rate $(133 \mathrm{mhz})$ laser ablation of aluminum with 1.2-ps pulses," Appl. Phys. A 69, S883-S886 (1999).

12. C. Kerse et al., "Ablation-cooled material removal with ultrafast bursts of pulses," Nature 537, 84-88 (2016).

13. H. Kalayciouglu, Y. B. Eldeniz, O. Akcaalan, S. Yavas, K. Gürel, M. Efe, and F. O. Ilday, "1 mj pulse bursts from a yb-doped fiber amplifier," Opt. Lett. 37, 2586-2588 (2012).

14. H. Kalaycioglu, K. Eken, and F. O. Ilday, "Fiber amplification of pulse bursts up to $20 \mu \mathrm{j}$ pulse energy at $1 \mathrm{khz}$ repetition rate," Opt. Lett. 36, 3383-3385 (2011).

15. H. Kalaycıoglu, P. Elahi, O. Akcaalan, and F. O. Ilday, "High-repetitionrate ultrafast fiber lasers for material processing," IEEE J. Sel. Top. Quantum Electron. 24, 1-12 (2018).

16. S. Breitkopf, A. Klenke, T. Gottschall, H.-J. Otto, C. Jauregui, J. Limpert, and A. Tünnermann, "58 mj burst comprising ultrashort pulses with homogenous energy level from an yb-doped fiber amplifier," Opt. Lett. 37, 5169-5171 (2012).

17. H. Yu, J. Zhang, Y. Qi, L. Zhang, S. Zou, L. Wang, and X. Lin, "85-w burst-mode pulse fiber amplifier based on a q-switched mode-locked laser with output energy $0.5 \mathrm{mj}$ per burst pulse," J. Light. Technol. 33, 1761-1765 (2015).

18. N. Jiang, M. C. Webster, and W. R. Lempert, "Advances in generation of high-repetition-rate burst mode laser output," Appl. Opt. 48, B23-B31 (2009).

19. P. Wang and W. A. Clarkson, "High-power, single-mode, linearly polarized, ytterbium-doped fiber superfluorescent source," Opt. Lett. 32, 2605-2607 (2007).

20. N. Finlayson, B. K. Nayar, and N. J. Doran, "Switch inversion and polarization sensitivity of the nonlinear-optical loop mirror," Opt. Lett. 17, 112-114 (1992).

21. G. P. Agrawal, Nonlinear fiber optics (Academic Press, San Diego, 2007).

22. C. B. Clausen, J. H. Povlsen, and K. Rottwitt, "Polarization sensitivity of the nonlinear amplifying loop mirror," Opt. Lett. 21, 1535-1537 (1996).

23. R. R. Gattass, L. R. Cerami, and E. Mazur, "Micromachining of bulk glass with bursts of femtosecond laser pulses at variable repetition rates," Opt. Express 14, 5279-5284 (2006).

24. I. Will, H. I. Templin, S. Schreiber, and W. Sandner, "Photoinjector drive laser of the flash fel," Opt. Express 19, 23770-23781 (2011).

25. B. Thurow, J. Hileman, W. Lempert, and M. Samimy, "A technique for real-time visualization of flow structure in high-speed flows," Phys. Fluids 14, 3449 (2002).

26. P. Wu, W. L. Lempert, and R. B. Miles, "Megahertz pulse-burst laser and visualization of shock-wave/boundary-layer interaction," AIAA J. 38, 672-679 (2000). 